\title{
Disappearance of Bone Marrow Fibrosis in a Patient with Chronic Myeloid Leukemia Treated with Dasatinib
}

\author{
Ernesto Vigna $^{a}$ Bruno Martino $^{c}$ Francesco Bacci $^{d}$ Anna Grazia Recchia ${ }^{e}$ \\ Francesco Mendicino $^{a}$ Rosellina Morelli ${ }^{b}$ Francesca Romana Mauro $^{f}$ Caterina Musolino ${ }^{g}$ \\ Rosa Greco $^{a}$ Eugenio Lucia ${ }^{a}$ Elena Sabattini ${ }^{d}$ Fortunato Morabito ${ }^{a}$ e Massimo Gentile ${ }^{a}$ \\ ${ }^{a}$ Hematology Unit and ${ }^{b}$ Nuclear Medicine Unit, Department of Onco-Hematology, AO of Cosenza, Cosenza, \\ 'Hematology Unit, Azienda Ospedaliera Bianchi Melacrino Morelli, Reggio Calabria, ${ }^{\mathrm{d}}$ Hemolymphopathology Unit, \\ Department of Hematology and Oncology "L \& A Seragnoli", S. Orsola-Malpighi Hospital, University of Bologna,

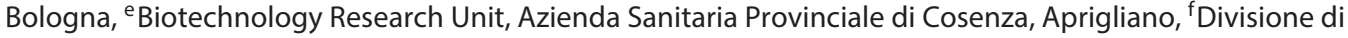 \\ Ematologia, Università La Sapienza, Rome, and ${ }^{9}$ Division of Hematology, University of Messina, Messina, Italy
}

\section{Established Facts}

- Dasatinib is a second-generation tyrosine kinase inhibitor with demonstrated clinical activity in all stages of chronic myeloid leukemia. It is a dual SRC/ABL kinase inhibitor, is 325 -fold more potent against BCR-ABL than imatinib, and has significant inhibitory activity against wild-type c-KIT and platelet-derived growth factor.

\section{Novel Insights}

- Dasatinib therapy resulted in a rapid disappearance of bone marrow fibrosis in a patient with chronic myeloid leukemia.

\section{Keywords}

Chronic myeloid leukemia · Myelofibrosis · Dasatinib tains a complete cytogenetic response and a deep molecular response; the last bone biopsy confirmed the absence of fibrosis.

(c) 2017 S. Karger AG, Basel

\begin{abstract}
We report a case of a chronic myeloid leukemia patient showing progressive bone marrow fibrosis and anemia during imatinib therapy. Given the loss of major molecular response, we switched treatment to dasatinib $100 \mathrm{mg}$ daily, observing a reduction in $\mathrm{BCR}-\mathrm{ABL}$ transcript, a significant improvement of anemia, and a gradual disappearance of fibrosis. After 7 years of dasatinib therapy the patient main-
\end{abstract}

\section{KARGER}

E-Mail karger@karger.com

www.karger.com/che
(C) 2017 S. Karger AG, Basel

\section{Introduction}

Chronic myeloid leukemia (CML) is a clonal myeloproliferative disorder that arises from a reciprocal balanced translocation between the long arms of chromosomes 9 and 22: $t(9 ; 22)$. The resulting Philadelphia chromosome 


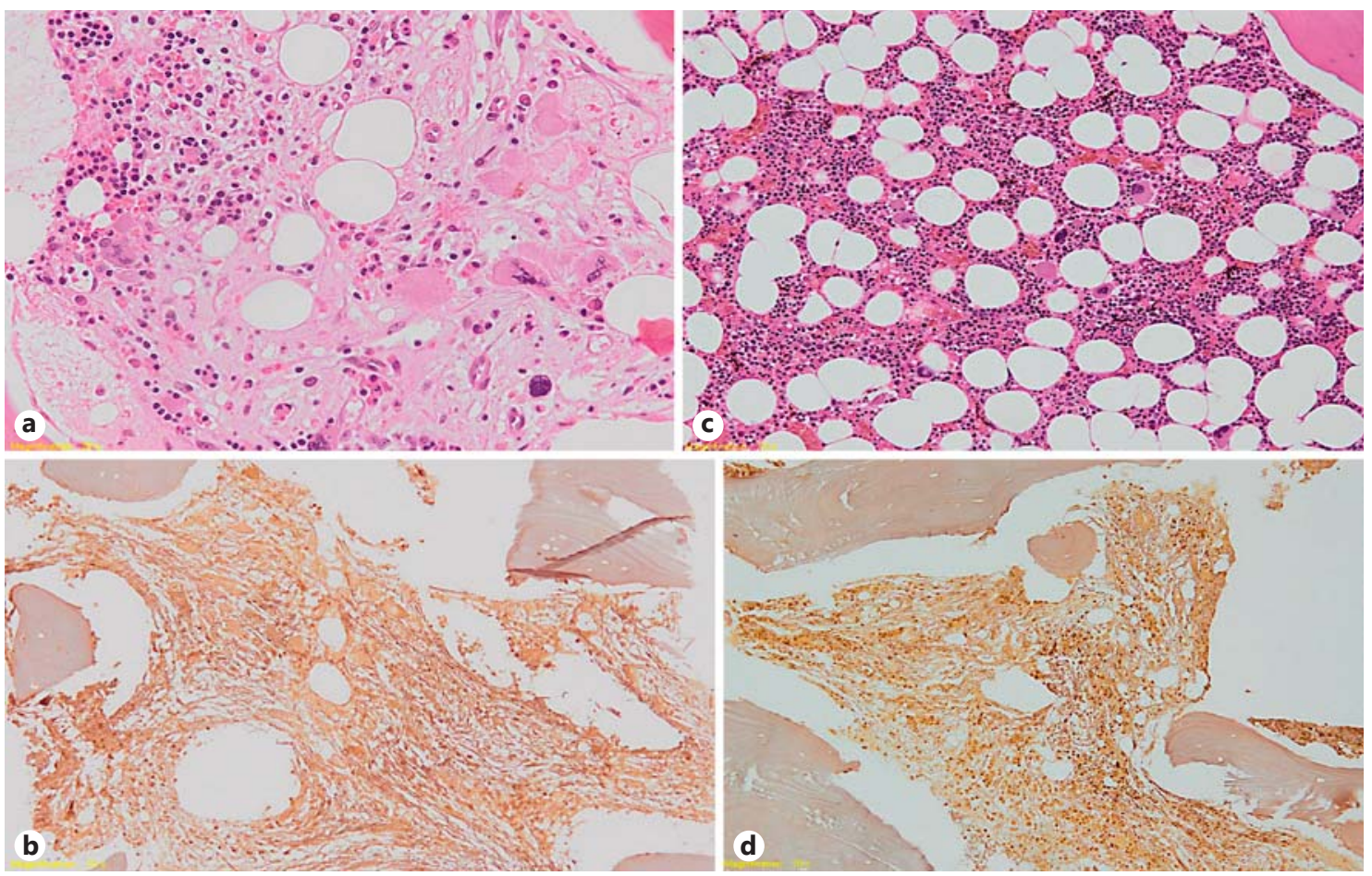

Fig. 1. Chronic myeloid leukemia before $(\mathbf{a}, \mathbf{b})$ and after $(\mathbf{c}, \mathbf{d})$ dasatinib therapy. $\mathbf{b}$ The silver stain illustrates an increase in the reticulin fibers. $\mathbf{d}$ The corresponding stain after 8 months of dasatinib therapy shows a reduction of fibrosis.

gives rise to the BCR-ABL fusion protein, a constitutively active tyrosine kinase that drives survival and proliferation and is responsible for leukemogenesis [1]. Treatment for CML is based on the use of the first-generation tyrosine kinase inhibitor (TKI), imatinib mesylate, and secondgeneration TKIs nilotinib and dasatinib, which have dramatically changed the natural history of the disease over the last 10 years [1]. Cytokines of the fibrosis-related network and biological abnormalities may be evident at the onset and during the clinical course of the disease. The presence of severe bone marrow fibrosis diagnosed at disease onset is associated with worse prognosis [2]. In CML, imatinib may reverse marrow fibrosis but does not consistently avoid its de novo development [3]. We describe herein a case of a CML patient receiving imatinib mesylate, showing a progressive increase of marrow fibrosis, which totally resolved after switching to dasatinib.

\section{Case Report}

A 45-year-old male patient was admitted to our hematology department in 1995 with severe leukocytosis. A bone marrow biopsy revealed hypercellular bone marrow, with an increased ratio of myeloid/erythroid precursors, compatible with chronic myeloprolif- erative disorder. The bone marrow cytogenetic analysis revealed the presence of the Philadelphia chromosome in all metaphases, and the molecular examination was positive for the BCR-ABL transcript. A low-risk Sokal CML diagnosis was made and, in the preTKI era, therapy with interferon was started. During interferon therapy the patient achieved a complete hematological response associated with a reduction of the BCR-ABL transcript after 1 year. However, he never reached a major molecular response (MMR). Therefore, in October 2000, with the introduction of the first-generation TKI, the patient began treatment with imatinib $400 \mathrm{mg} /$ day, obtaining an MMR after the first 3 months of therapy. However, in October 2006, the patient presented a progressive anemia, with a minimum hemoglobin $(\mathrm{Hb})$ value of $7 \mathrm{~g} / \mathrm{dL}$. BCR-ABL monitoring still revealed an MMR associated with a complete cytogenetic response (CCyR). The patient started a-erythropoietin treatment at a weekly dose of 40,000 UI. Hb levels showed a progressive increase up to a maximum value of $11 \mathrm{~g} / \mathrm{dL}$. In May 2008, the patient experienced a new episode of severe anemia $(\mathrm{Hb} 8 \mathrm{~g} / \mathrm{dL}$ ), together with an on log increase of BCR-ABL transcript in the bone marrow (BCR-ABL ${ }^{\mathrm{IS}} \%$ from 0.011 to 0.118 ), while he maintained a CCyR. The imatinib dose was increased to $600 \mathrm{mg} /$ day. Due to the persistence of anemia ( $\mathrm{Hb} 9 \mathrm{~g} / \mathrm{dL}$ ), in November 2008 the patient underwent a bone marrow biopsy, which clearly showed a fibrotic pattern (Fig. 1a, b). Cytogenetic analysis was negative for the presence of Philadelphia chromosome and/or other cytogenetic abnormalities, with a stable BCR-ABL transcript (BCR-ABL $\%=0.199$ ). In January 2009 , a worsening of anemia $(\mathrm{Hb} 7 \mathrm{~g} / \mathrm{dL}$ ) was observed, which required red blood cell support. Notably, an increase in BCR-ABL transcript levels was documented $\left(\mathrm{BCR}-\mathrm{ABL}^{\mathrm{IS}} \%=1.34\right)$ with loss 
of MMR. FISH analysis showed a reappearance of the Philadelphia chromosome (2 out of 20 metaphases, 10\%) with the loss of CCyR. Thus, we switched treatment to dasatinib $100 \mathrm{mg}$ daily. A significant improvement of anemia was achieved after 3 months of dasatinib therapy, and the patient became transfusion free; moreover, a reduction in BCR-ABL transcript was achieved $\left(\mathrm{BCR}-\mathrm{ABL}^{\mathrm{IS}} \%=\right.$ 0.34 ). After 12 months of dasatinib treatment, bone marrow histology revealed a reduction of fibrosis (Fig. 1c, d). Molecular and cytogenetic evaluation showed a further reduction of the BCR-ABL transcript $\left(\mathrm{BCR}-\mathrm{ABL}^{\mathrm{IS}} \%=0.08\right)$ and a CCyR. Bone marrow biopsy was repeated annually and showed a gradual disappearance of fibrosis. Currently, the patient maintains a deep molecular response and CCyR and continues dasatinib treatment. The last bone biopsy, performed in June 2016, confirmed the absence of fibrosis.

\section{Discussion}

Since histopathology of the bone marrow had never been considered in the management of CML, there is a lack of long-term information about bone marrow histology and its variation during therapy. Bone marrow fibrosis in CML does not seem to be a rare event. It can be found in the bone marrow of about $30-40 \%$ of patients before treatment and has been shown to be associated with adverse prognosis, independently of other prognostic factors [2]. In fact, the worsening of bone marrow fibrosis during therapy is an early indicator of disease progression, which precedes the changes in the peripheral blood, the spleen size, or marrow blast counts [2]. To date, the exact pathophysiology of bone marrow fibrosis in CML is unknown, but it is believed to be secondary to cytokine release (transforming growth-factor- $\beta$ and platelet-derived growth factor, PDGF) by megakaryocytes and to an abnormal stimulation of fibroblasts [2]. Some studies have shown the ability of imatinib to induce a significant regression of bone marrow fibrosis in CML patients, which was paralleled by a marked decrease of megakaryocytes and a specific anti-PDGF effect, but was not correlated with a cytogenetic response $[3,4]$. In fact, imatinib is not only an inhibitor of ABL, but was originally developed as a specific PDGF receptor inhibitor [5]. However, imatinib does not guarantee against the evolution of marrow fibrosis and showed modest activity in patients with primary myelofibrosis [6]. Dasatinib is a second-generation TKI with demonstrated clinical activity in all stages of CML; it is a dual SRC/ABL kinase inhibitor, is 325-fold more potent against BCR-ABL than imatinib, and has significant inhibitory activity against wild-type c-Kit and PDGF [7]. SRC kinases play important roles in the activation of fibroblasts and in the development of fibrosis [8].

Furthermore, since BCR-ABL seems to be implicated in the phosphorylation of STAT5, another hypothesis regarding the regression of marrow fibrosis is that dasatinib inhibits the JAK/STAT5 cascade that represents the classical pathway for marrow fibrosis treatment [9].

In our patient, bone marrow fibrosis anticipated the loss of both the molecular and cytogenetic responses during treatment with imatinib, confirming its role as an early indicator of disease evolution. Dasatinib therapy resulted in a rapid disappearance of fibrosis, suggesting its higher efficacy compared to imatinib. In fact, the ability to inhibit both SRC kinase and PDGF gives dasatinib a more potent antifibrotic effect. In our case, considering the platelet count settled between 150 and $250 \times 10^{9} / \mathrm{L}$ during the course of CML, it is unlikely that there was any relationship with bone marrow fibrosis. These findings offer novel therapeutic possibilities in diseases with abnormal bone marrow fibrosis.

\section{Disclosure Statement}

The authors report no conflicts of interest.

\section{References}

1 Rosti G, Castagnetti F, Gugliotta G, Baccarani M: Tyrosine kinase inhibitors in chronic myeloid leukaemia: which, when, for whom? Nat Rev Clin Oncol 2017;14:141-154.

2 Buesche G, Hehlmann R, Hecker $\mathrm{H}$, et al: Marrow fibrosis, indicator of therapy failure in chronic myeloid leukemia - prospective long-term results from a randomized-controlled trial. Leukemia 2003;17:2444-2453.

3 Bueso-Ramos CE, Cortes J, Talpaz M, et al: Imatinib mesylate therapy reduces bone marrow fibrosis in patients with chronic myelogenous leukemia. Cancer 2004;101:332-336.
4 Beham-Schmid C, Apfelbeck U, Sill H, et al: Treatment of chronic myelogenous leukemia with tyrosine kinase inhibitor STI571 results in marked regression of bone marrow fibrosis. Blood 2002;99:381-383.

5 Buchdunger E, Cioffi L, Law N, et al: Abl protein-tyrosine kinase inhibitor STI571 inhibits in vitro signal transduction mediated by c-Kit and platelet-derived growth factor receptors. J Pharmacol Exp Ther 2000;295:139-145.

6 Tefferi A, Mesa RA, Gray LA, et al: Phase 2 trial of imatinib mesylate in myelofibrosis with myeloid metaplasia. Blood 2002;99: 3854-3856.
7 Talpaz M, Shah NP, Kantarjian H, et al: Dasatinib in imatinib-resistant Philadelphia chromosome-positive leukemias. N Engl J Med 2006;354:2531-2541.

8 Skhirtladze C, Distler O, Dees C, et al: Src kinases in systemic sclerosis: central roles in fibroblast activation and in skin fibrosis. Arthritis Rheum 2008;58:1475-1484.

9 Gowin K, Emanuel R, Geyer H, Mesa RA: The new landscape of therapy for myelofibrosis. Curr Hematol Malig Rep 2013;8:325-332. 